\title{
Il restauro del castello di Massafra (TA)
}

\author{
The restoration of Massafra' Castle (TA)
}

\section{Tiziana de Gennaro ${ }^{a}$, Davide de Leo ${ }^{b}$}

${ }^{a}$ Ministero per i Beni e le Attività Culturali e per il Turismo, Rome, Italy, degennaro.tiziana@gmail.com

${ }^{\mathrm{b}}$ Politecnico di Bari, Terlizzi (Ba), Italy, dvd.deleo@gmail.com

\begin{abstract}
Massafra Castle's restoration project aims to preserve and enhance the monument, redeeming it from the current state of partial abandonment, with the scope to return it to the community. First, an in-depth study of an historical research was carried out together with the analysis of the monument' superficial and structural degradation to identify the adequate remedies. It was clear that "restoring only the stones" would have not been efficient and therefore finding a new purpose was necessary and essential. Finding a new scope for those kinds of architectures is a matter of great interest, because more than any other type of monuments, those are completely out of their historical context that gave them political, military and economic reason to be built.

Therefore, following a sociological investigation, it emerged that the best solution would be create a connection between Massafra and cinematography: there are many cultural organizations in this area and most of them need more dedicated space. Massafra has already been chosen several times as a movie set by famous authors: Il Vangelo secondo Matteo by Pier Paolo Pasolini, Il Paese delle spose infelici by Pippo Mezzapesa and Amiche da morire by Giorgia Farina. This is how the MOVIE (Massafra Omni Vision between Innovation and Cultural Heritage) idea was born, a $360^{\circ}$ cinema setting which is unique at a national level and that it would become part of larger cinematographic circuit already flourishing in the Apulia region. The project's feasibility was then evaluated from a logistic-economic point of view. Furthermore, given the Castle's location, the project proposes the reopening of ancient paths that connect the monument to the historical town centre and to the Ravine. The project also supports the restoration of rock settlements with its cave houses (currently completely collapsed) that surround the castle's foundation to divulge hospitality.
\end{abstract}

Keywords: Harnessing, rifunzionalizzazione, sociology, cinematography.

\section{Introduzione}

Il presente articolo è elaborazione di parte di una più ampia ricerca di tesi di laurea condotta dagli architetti Francesco Cardone, Tiziana de Gennaro, Davide De Leo, Giuseppe De Marinis Gallo, Maria Anna De Palma e Silvia Manginelli, discussa nel 2013 presso il Politecnico di Bari con il coordinamento della Professoressa Rossella de Cadilhac.

L'ampio lavoro di ricerca effettuato si concentrerà, in questo contesto, sull'importanza di trovare una destinazione d'uso compatibile con il monumento. Il riuso, infatti, sebbene non debba essere considerato come fine del restauro, assume un'importanza fondamentale se visto nell'ottica di mezzo con il quale perpetrare la conservazione dei monumenti. 
Il progetto di restauro del Castello di Massafra si prefigge infatti l'obiettivo di riscattare il monumento dall'attuale stato di parziale abbandono in cui versa, per restituirlo alla collettività e trasmetterlo alle generazioni future, esprimendo con pienezza i valori singolari ed irripetibili di Bene Culturale che gli sono propri, cioè di "testimonianza materiale avente valore di civiltà"1.

Inoltre il castello risulta elemento di connessione fra la gravina di San Marco a sud-est, dove si conservano i segni di un antico villaggio rupestre, e l'antico tessuto urbano a nord-ovest.

Nello spirito della Dichiarazione di Amsterdam e delle più recenti Carte del Restauro, si ritiene opportuno estendere la valorizzazione del castello anche al contesto ambientale del quale il manufatto è parte integrante ed al quale si riconosce un notevole valore paesaggistico (Fig. 1).

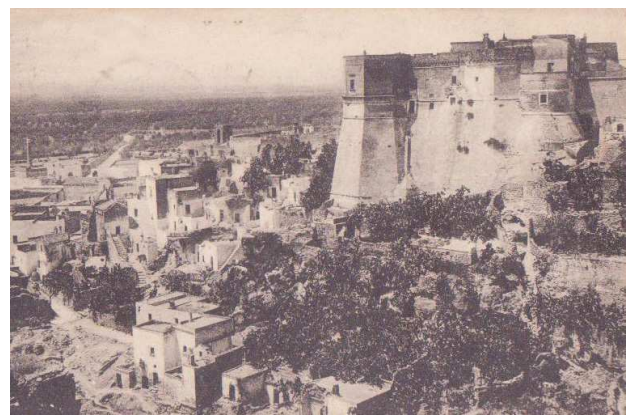

Fig. 1. Lo spalto occidentale della gravina di San Marco nel 1960.

Da questo assunto prende forma l'intento di conservare e valorizzare i caratteri identitari del castello, da quello monumentale a quello paesistico, anche riconnettendo i rapporti che esso ha avuto, nel corso della tempo, con il nucleo antico della città e con la gravina, interrotti successivamente a causa del sopraggiungere di calamità naturali e modifiche di natura antropica.

Ai fini progettuali è stato indispensabile lo studio approfondito dell'opera, mediante il rilievo ed il riconoscimento delle sue principali fasi costruttive. Ciò ha permesso di individuare e comprendere le ragioni che hanno definito l'attuale articolazione spaziale e le specificità costruttive del castello intervenendo con un approccio ri- spettoso dell'identità e della storia del bene culturale da tutelare.

I criteri della riconoscibilità, reversibilità e del minimo intervento, a garanzia del rispetto della materia antica e dell'espressività degli interventi attuali, mirano a restituire l'unità di immagine laddove si riscontrano lacune di parti storicamente accertate, come ad esempio per gli ambienti la cui spazialità è compromessa.

La completa valorizzazione del monumento, come detto, non può prescindere dalla scelta di una destinazione d'uso, in linea con l'invito espresso dalla Dichiarazione di Amsterdam ad una "conservazione integrata".

\subsection{Contesto e inquadramento storico}

L'ubertosità del territorio massafrese, caratterizzato da una triplice geografia, in sequenza pianura, colline e boschi verso nord, l'abbondanza di risorse naturali e la presenza delle gravine costituirono, in passato, il motivo che rese Massafra un importante nucleo urbano nella storia medioevale e sono tutt'ora delle invarianti strutturali, legami profondi tra cultura materiale e modi d'uso umani delle risorse ambientali (Fig. 2).

Numerosi reperti risalenti al periodo neolitico, ritrovati nell'insediamento rupestre di S. Maria della Scala, a nord dell'attuale abitato di Massafra, lasciano supporre che già dal Neolitico vi sia stata continuità della presenza umana, dapprima stabilitasi in grotte naturali e successivamente stanziata in rifugi ricavati per scavo nella roccia detti "vicinanze" (Jacovelli, 1981, pp. 8-9).

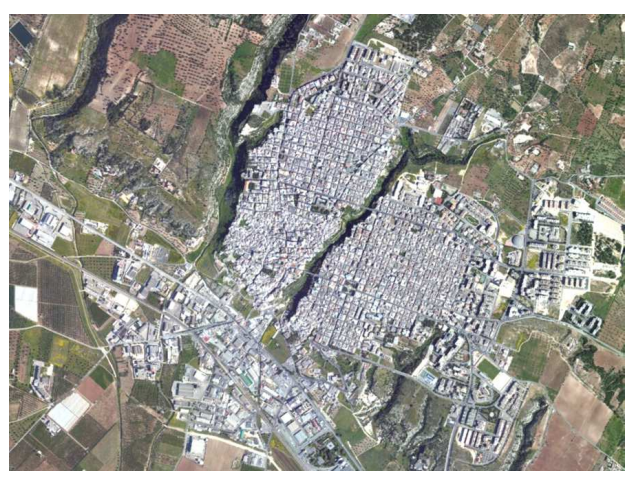

Fig. 2. Massafra solcata dalle gravine. 
Le origini del castello risalgono probabilmente all'XI secolo, ossia durante la dominazione normanna della città ${ }^{2}$.

Come molti dei castelli presenti nel sud Italia, ed in particolare appartenenti alla "Settentrionale Ionica", l'evoluzione formale e spaziale si lega intimamente alla successione di dominazioni passando da mani normanne al dominio svevo, durante il quale fu rimesso in efficienza da Federico II (Sthamer, 1995, pp. 94-150). Nel 1269 il castello divenne residenza del feudatario di Carlo I d'Angiò, Oddone de Soliac (Guerrieri, 1900, p. 81).

Attraverso l'analisi delle tessiture murarie, della configurazione planimetrica ed il confronto con castelli del circondario si può supporre che, durante il periodo Angioino, il castello si presentasse con un impianto pressoché quadrangolare con al centro un cortile caratterizzato da arcate ogivali, tutt'ora presenti.

Sotto il dominio Aragonese nel 1497 si insediò nel feudo la nobile famiglia del Pappacoda che trasformarono il castello da edificio fortificato a dimora signorile (Gallo, 1916, pp. 70-71) mediante l'adeguamento delle torri circolari, la costruzione delle scarpate sulle murature ed un primo coronamento.

A questo periodo risale sicuramente anche la torre ottagonale ${ }^{3}$.

Dal 1622 il potere della famiglia napoletana entrò in crisi, e nel 1633 il feudo di Massafra fu venduto alla famiglia Carmignano che, intorno al 1661, la cedette alla famiglia Imperiali (Jacovelli, 1983, p. 33). Questi trasformarono il castello in una piccola reggia con nuove finestre, volte, merlature, l'elevazione della torre ottagonale e la realizzazione di una monumentale rampa d'accesso alla piazza d'armi, per superare il dislivello tra piano cortile e il piano strada (Cazzato, Basile, 2008, pp. 72-91). Anche l'abitato si espanse fino a raggiungere i confini che resteranno stabiliti per circa un secolo e mezzo, e che oggi costituiscono i limiti del centro storico.

A seguito della soppressione del feudalesimo il Castello di Massafra fu acquistato dal ricco possidente tarantino Cataldo Zuccaretti (Jacovelli, 1971). Alla morte di quest'ultimo, nel 1859, il castello passò nelle mani degli eredi, che vi apportarono diverse modificazioni al fine di adattarlo a residenza per più famiglie.

Nel 1961 il Castello, concesso in uso al Comune, fu in parte trasformato in scuola, subendo pesanti manomissioni, dovute all'installazione degli impianti necessari e all'introduzione di svariate tramezzature nelle stanze al piano nobile.

Il 12 febbraio 1965 crollò il torrione ovest a causa dell'eccessiva imbibizione del nucleo murario e qualche mese dopo Piero Gazzola, presidente dell'istituto Nazionale dei Castelli, nonché partecipe estensore della Carta di Venezia, segnalava, alla Soprintendenza dei Beni Ambientali Architettonici Artistici e Storici della Puglia, l'urgenza di un intervento radicale mediante, in primis, il riconoscimento di monumento nazionale, la ricostruzione della torre crollata ed il restauro dell'intero monumento al fine di destinarlo a museo civico e pinacoteca.

Dopo l'acquisto del compendio da parte dell'amministrazione comunale nel 1976, iniziarono i primi interventi di consolidamento delle murature, a cura dell'Ing. De Tommasi e dell'Arch. Benedettelli, mediante iniezioni armate e cerchiatura del torrione nord. Nel 1985 a causa di infiltrazioni d'acqua e del peso di un autocarro colmo di materiali di risulta, si verificò la frana di una porzione della piazza d'armi nelle vicinanze della torre ottagonale che coinvolse anche le abitazioni in grotta sottostanti che furono completamente distrutte (Fig. 3).

Dal 1985 al 1997 furono eseguiti due interventi di restauro diretti dall'Arch. Mauro Civita di ricostruzione del torrione crollato, consolidamento globale delle fondazioni, rimozione delle parti pericolanti, restauro dell'ala sud-ovest per la realizzazione della biblioteca comunale al piano primo e del museo archeologico e dell'olio e del vino al piano terra.

Ad oggi all'incirca più della metà della superficie del castello è in uno stato di abbandono; sono regolarmente funzionanti, come biblioteca, solo dieci ambienti del piano nobile del castello, nell'ala sud, comprese le due torri. Le sale che ospitano il museo dell'olio e del vino e le due sa- 
le che conservano i reperti archeologici sono in genere chiuse al pubblico.

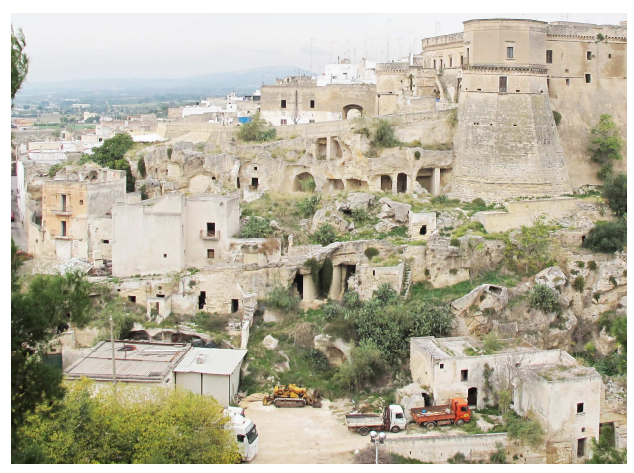

Fig. 3. Resti dell'agglomerato di case-grotta ai piedi del Castello (Silvia Manginelli 2012).

\section{La scelta della destinazione d'uso}

Si è manifestata la necessità di attuare una scelta progettuale coerente che si integrasse con la biblioteca comunale prendendo le distanze, tuttavia, dalla funzione museale decisamente estranea alla cittadinanza.

Un'attenta analisi effettuata sul territorio è stata di fondamentale importanza per la scelta della nuova destinazione d'uso che fosse coerente tanto con la vocazione funzionale del Castello quanto con le esigenze della popolazione massafrese. Solo partendo dall'individuazione dei fabbisogni territoriali e della risposta a tali fabbisogni è stato possibile stabilire se l'intervento fosse effettivamente fattibile e necessario o meno.

Si è effettuata un'indagine della domanda a scala locale, che tenesse conto delle esigenze della cittadinanza, per poi estendere il raggio d'azione delle ricerche a livello regionale e nazionale.

Sono stati sottoposti alla cittadinanza dei questionari $^{4}$ da cui si è rilevato che $\mathrm{i}$ giovani e gli adulti non hanno l'abitudine di frequentare il centro storico poiché considerato un luogo degradato e, quasi la totalità degli intervistati, considerano il castello simbolo indiscusso della città e ne vorrebbero il recupero e la rifunzionalizzazione, soprattutto per attività inerenti il teatro e il cinema.
Dall'esame delle 79 associazioni presenti sul territorio, si è rilevato che più del $49 \%$ è operante nel settore culturale. Particolarmente attive sono le associazioni che si occupano di arti visive e utilizzano il cinema come mezzo di approfondimento delle tematiche sociali e di valorizzazione del territorio. Ogni anno Massafra è, infatti, protagonista di un festival cinematografico, arrivato alla XIV edizione e che, essendo ambientato nel centro storico, si prefigge anche l'obiettivo di sensibilizzare la popolazione ed invogliarla alla frequentazione della città antica 5 .

Massafra è stata set cinematografico per diversi film, tra cui, Il Vangelo secondo Matteo di Pier Paolo Pasolini ${ }^{6}$, Il paese delle spose infelici di Pippo Mezzapesa e Amiche da morire di Giorgia Farina.

Considerati i bisogni e le necessità espressi dalla cittadinanza massafrese e dalle associazioni il progetto di rifunzionalizzazione parte quindi dall'idea di far confluire le varie realtà disomogenee che si occupano delle attività nel settore teatrale e cinematografico in un unico complesso, un Polo del Cinema, in grado di rendere partecipi di tali attività tutti i componenti della popolazione. Il Castello, in quanto bene culturale simbolo della città e data la sua collocazione strategica rispetto al centro cittadino ed alle infrastrutture di collegamento extra-urbane, si configurerebbe come il luogo più idoneo ad ospitare iniziative che coinvolgano la comunità.

Il Polo Cinematografico diventerebbe un luogo di insegnamento, ricerca e sperimentazione nel campo della cinematografia attraverso la realizzazione al proprio interno di laboratori aperti al pubblico, gestiti dalle associazioni locali e attualmente distribuite in maniera disomogenea all'interno di locali in affitto.

Anche a livello regionale si sono rilevate necessità legate al settore cinematografico. In particolare, alcuni collezionisti pugliesi di pellicole necessitano la creazione di una Cineteca Regionale in grado di dare una più idonea collocazione alle loro collezioni; l'associazione Apulia Film Commission, nata nel 2007, ha stanziato fondi per la produzione di film in Puglia, generando un afflusso di troupe cinematografiche, a seguito 
del quale è sorta l'esigenza di creare centri specializzati nella post produzione cinematografica.

A livello nazionale la domanda è stata espressa dall'assenza di poli cinematografici nel Sud Italia e dalla necessità di archiviare il materiale prodotto in Puglia a seguito della fondazione della Apulia Film Commission sotto l'esempio della Cineteca di Roma.

Il progetto del MOVIE (Massafra Omni Vision tra Innovazione ed Eredità culturale) si articola in un centro di post produzione, una cineteca e la sede delle diverse associazioni massafresi che porterebbe non solo alla rivalutazione del centro storico, ma inserirebbe anche l'area tarantina nel circuito cinematografico regionale.

Da tale ipotesi sono state escluse quelle funzioni che potessero ledere la struttura per necessità di impiantistica particolare o che esigessero grandi spazi non presenti nel Castello.

Lo studio dell'offerta, volto ad individuare i possibili competitors ha rilevato la mancanza di una realtà simile nel Sud Italia ${ }^{7}$, non esistendo poli cinematografici che possano soddisfare in pieno e su tutti i fronti la domanda presente sul territorio.

Oltre a ciò, la grande affluenza, negli ultimi anni, di troupe cinematografiche nel Meridione permette di definire ulteriori funzioni del progetto atte all'accoglienza e basi operative per il lavoro di post produzione cinematografica. La realizzazione di poli di questo tipo potrebbe funzionare da catalizzatore per attrarre le troupe che scelgono la Puglia come set cinematografico a realizzarvi i lavori di ripresa e tutta la fase successiva di postproduzione.

Il ristabilimento delle perdute connessioni tra castello e gravina ed il restauro delle case-grotta ormai distrutte costituiscono la perfetta occasione per la creazione di un albergo diffuso, sul modello del più famosi Sassi di Matera, che possa pertanto accogliere le funzioni di cui sopra.

\subsection{Il progetto di restauro e la rifunzionaliz- zazione degli spazi}

La proposta di rifunzionalizzazione prevede quindi la creazione un complesso polifunzionale legato alla sfera cinematografica (centro di postproduzione cinematografico, sala cinema e conferenze, sede di associazioni culturali, mediateca, biblioteca, archivio pellicole) che restituirà al monumento una nuova vita e risponderà alle necessità sociali e culturali non solo della città massafrese ma anche della Puglia.

Le necessarie opere di consolidamento prevedono l'utilizzo di fasciature esterne e interne alla torre ovest in fibre aramidiche, delle volte degli ambienti del primo e secondo livello, unitamente a collegamenti trasversali dei paramenti murari tramite barre nello stesso materiale. Al fine di agevolare il drenaggio e l'evaporazione dell'acqua di cui le murature risultano imbibite, saranno praticati dei fori in profondità e inclinati lungo tutto l'apparato murario che saranno inettati di malta compatibile con le caratteristiche chimico-fisica di quella esistente, una volta che il nucleo delle murature risulterà asciutto.

il trattamento delle superfici interne sarà effettuato mediante intonaco per le murature e le volte e scialbatura per gli ambienti che non presentano tracce di intonaci antichi. Per quel che concerne le superfici esterne, si è deciso di non ripristinare integralmente gli intonaci al fin di non alterare l'immagine del monumento ormai consolidata. I lacerti di intonaco in disgregazione saranno trattati con l'applicazione di materiale consolidante organico che ristabilirà coesione fra le particelle di materiale e ri-adesione dei vari strati al supporto murario. Saranno integrate solo piccole porzioni di intonaco, limitandosi agli strati più interni (ex. arriccio), accordando la cromia con quella esistente mediante l'aggiunta di terre naturali all'impasto. Per paramenti a facciavista si avrà cura di integrare le lacune con conci di litotipo analogo a quello esistente, ristilare dei giunti e stuccare le fessure.

Alla quota più bassa del complesso, ossia quella della piazza d'Armi, è riabilitato il portale settecentesco, che attualmente svolge la funzione di accesso secondario, mediante la sostituzione del cancello esistente con uno in acciaio corten.

In un ambiente del piano interrato è prevista anche una piccola sala cinema. 

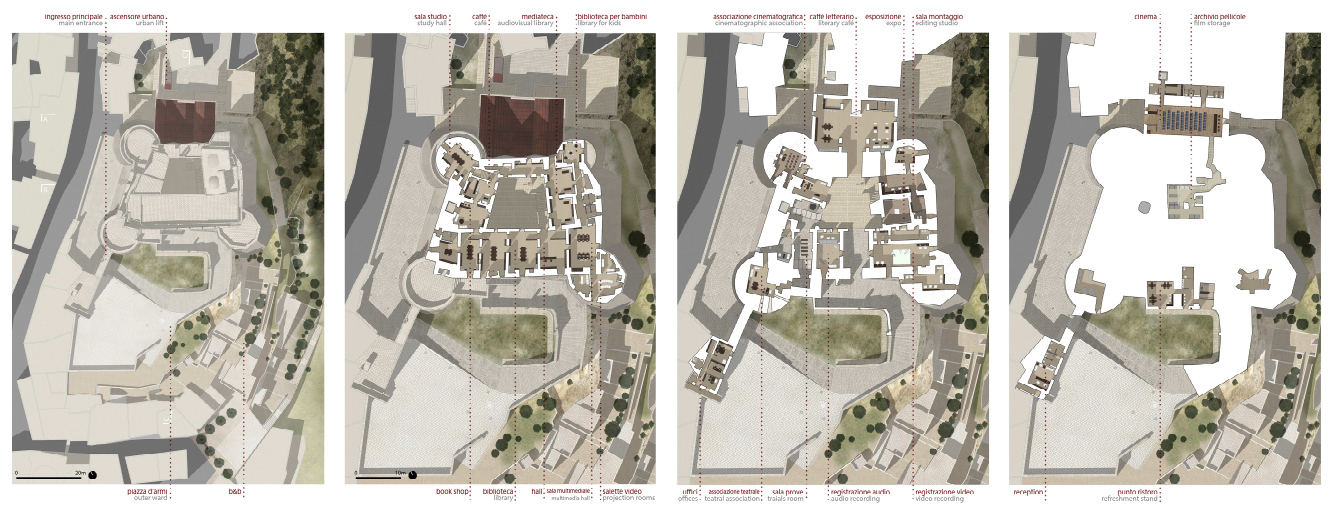

Fig. 4. Il Progetto funzionale.

Il centro di postproduzione cinematografico è situato al piano terra del castello, le sedi di associazioni culturali massafresi, sono collocate dove erano presenti le antiche stalle, e un piccolo spazio museale, dedicato alla figura cinematografica di Pier Paolo Pasolini, è ubicato nella cappella di san Lorenzo.

Alle attività di natura culturale si associa la creazione di un Caffè letterario e di punti ristoro aperti a tutta la cittadinanza in grado di rendere vissuto il monumento alle diverse ore del giorno.

Il Caffè letterario trova spazio nei magazzini, che attualmente costituiscono l'entrata principale al castello; qui sono ripristinate le coperture a capriata, andate perdute, la cui fisionomia è stata ricostruita tramite le tracce esistenti, mentre per ciò che concerne i materiali, esse sono realizzata in legno e corten, prevedendo anche la presenza di lucernari.

Nella corte interna sono rimosse le tamponature delle arcate ogivali e la pavimentazione incongrua, sostituendola con lastre di pietra a filari paralleli tessuti secondo la linea di massima pendenza.

Il piano nobile ospita la mediateca/biblioteca, dotata di sale multimediali. Al fine di organizzare al meglio i percorsi, sono ripristinate le aperture del 1961. La pavimentazione incongrua esistente è sostituita da una malta a cocciopesto, non avendo alcun dato storico riguardante la tessitura più antica (Fig. 4).

L'intervento, come detto, si propone di recuperare l'immagine che caratterizzava lo spalto occi- dentale della Gravina S. Marco $^{8}$ che costituiva un unicum paesaggistico, in cui natura ed architettura formavano un rapporto indissolubile.

Le volumetrie delle nuove cellule abitative ricalcano le forme planimetriche delle abitazioni antiche. Rispettando la tipologia insediativa antica si è progettata una facciata tecnologica a chiusura dello scavo che garantisse tutti gli standard abitativi minimi.

L'insediamento si svolge su differenti quote, collegate tra loro da un sistema di rampe e da scale trasversali, parallele ai muri d'ambito delle abitazioni; sono stati ricalcati i percorsi naturalistici già preesistenti in gravina $\mathrm{e}$, nel progettare quelli nuovi, si è fatto puntuale riferimento alla documentazione fotografica storica.

È stato anche progettato un sistema di risalita al castello che si compone di un ascensore urbano e di due rampe di scale realizzate in muratura, di cui una già preesistente (Fig. 5).

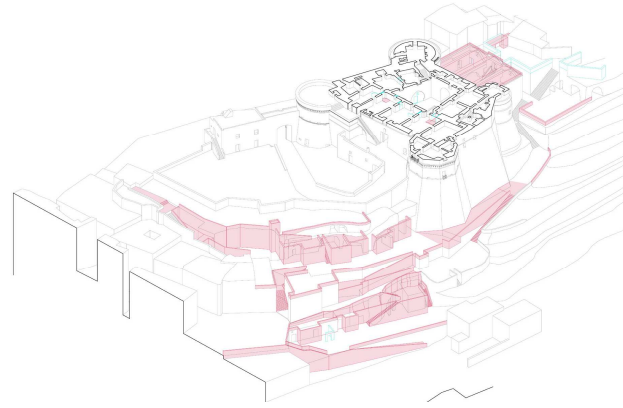

Fig. 5. Intervento di riconfigurazione paesaggistico. 


\section{Conclusioni}

L'analisi di fattibilità ha rivelato che il progetto, così come configuratosi, ha le capacità di autosostenersi e produce notevoli benefici per la collettività a cui afferisce. Le condizioni di funzionamento previste, inoltre, prefigurano un notevole beneficio non solo per tutta la cittadinanza ma, soprattutto, per il soggetto gestore.

L'intervento così realizzato, comporterebbe l'implementazione dell'offerta culturale creando nuove opportunità sotto differenti forme: valorizzazione del territorio e recupero del centro storico di Massafra dalle grandi e poco sfruttate potenzialità; recupero paesaggistico della gravina di San Marco; restauro architettonico del Castello; rilanci occupazionali e inserimento del bene all'interno di un circuito turistico e culturale già consolidato che partecipi alla crescita economica della città; promozione del territorio tramite la riproposizione della città di Massafra come set cinematografico e punto d'appoggio per le troupe; inserimento del bene all'interno di una rete di strutture che operano in campo cinematografico contribuendo alla crescita della Regione Puglia in tale ambito.

L'utilizzo continuativo del castello porterebbe sicuramente anche ad incrementare i cicli di manutenzione straordinaria. È doveroso ricordare che il restauro si configura sempre come un trauma per il monumento ed è solo tramite il mantenimento in efficienza dello stesso che tale trauma si può evitare.

\section{Note}

${ }^{1}$ Atti e documenti della Commissione di indagine per la tutela e la valorizzaione del patrimonio storico, archeologico, artistico e del paesaggio, per la salvezza dei beni culturali in Italia, Roma, Colombo Ed., 1967, p. 22.
${ }^{2}$ Archivio dell'Abbadia della SS. Trinità di Cava dei Tirreni (D,35 - 1100 Octobris, ind. 8, anno 14 ducatus Rogerii).

${ }^{3} \mathrm{Nel} 1599$ fu redatto un documento, pervenuto nell'Archivio di Stato di Napoli, nel quale il castello risulta già dotato di quattro torri, ponte d'accesso, camere per l'abitazione baronale ed altre di varia funzione.

${ }^{4}$ I questionari sono stati somministrati a campioni di popolazione di età omogenea, rispettando il rapporto numerico in base al genere.

${ }^{5}$ Tra le altre iniziative proposte dalle associazioni figurano anche rassegne cinematografiche per le scuole con laboratori sul linguaggio cinematografico, rassegne e retrospettive cinematografiche.

${ }^{6}$ Film del 1964 vincitore del Leone d'Argento Gran premio della giuria alla XXIV Mostra di Venezia, di tre Nastri d'Argento (migliore regia, migliore fotografia, migliori costumi) e nominato a tre Oscar (migliore scenografia, migliori costumi, migliore colonna sonora).

${ }^{7}$ A livello nazionale centri di eccellenza nel settore sono la Cineteche di Roma, Bologna e Milano. A scala territoriale si individuano la Cineteca Lucana che, ad oggi, presenta problemi organizzativi dovuti alla delocalizzazione in più sedi, e la Mediateca Regionale Pugliese, che si occupa della mera conservazione di documentazione audio visiva. Quest'ultima realtà potrebbe creare un binomio con una Cineteca regionale.

${ }^{8} \mathrm{Nel}$ progetto di ricostruzione si è costantemente fatto riferimento alla documentazione fotografica storica e ad una mappa catastale di Massafra datata 1930 ritrovata all'Archivio di Stato di Taranto.

\section{Bibliography}

Carbonara, G. (1997). Avvicinamento al restauro. Teoria, storia, monumenti, Liguori Ed., Napoli, pp. 371-383.

Cazzato, V.; Basile, V. (2008). Dal castello al palazzo baronale. Residenze nobiliari nel Salento dal XVI al XVII secolo, Congedo Mario Ed., Lecce, pp. 72-91.

Coscia, C.; Fregonara, E. (2004). Strumenti economico-estimativi nella valorizzazione delle risorse architettoniche e culturali, Celid Ed., Beinasco, pp. 19-25. 
Fiorani, D. (2004). Restauro Architettonico e strumento informatico, Liguori Ed., Napoli. pp. 116-119.

Fonseca, D.; Lembo, F. (1977). Centro storico di Massafra, Adriatica editrice SAL, Lecce, pp. 11-20.

Gallo, V. (1916). Origine e vicende della città di Massafra, Officina cromotipografica "aldina”, Napoli, pp. 70-140.

Guerrieri, F. (1900). Possedimenti temporali e spirituali dei Benedettini di Cava nelle Puglia, V. Vecchi Ed., Trani, pp. 29-81.

Jacovelli, E. (1971). Massafra nel sec. XVI, repertorio dei monumenti e delle opere d'arte, Edizioni della società operaia, Massafra, pp. 20-83.

Jacovelli, E. (1981). Massafra, la città e il territorio, Stampasud Posa, Mottola, pp. 5-17.

Jacovelli, E. (1983). Massafra nel sec. XVII, Edizioni Rettoria Madonna della Scala, Massafra. pp. 33-41.

Licino, R. (2010). Castelli Medievali. Puglia e Basilicata: dai Normanni a Federico II e Carlo d'Angiò, Caratteri mobili, Bari, pp. 22-113.

Marchesi, G. (2017). Valutazione economica di progetti di restauro e valorizzazione di risorse culturali, "L'Erma" di Bretschneider, Bari, pp. 66-72.

Marconi, P. (1986). "La questione delle superfici di sacrificio e le sue conseguenze metodologiche: il recupero critico delle tecniche tradizionali”, in Anastilosi. L'antico, il restauro, la città, Laterza Ed., Bari, pp. 192-199.

Mare; G. De; Nestico, A.; Caprino R.M. (2012). La valutazione finanziaria dei progetti per il rilancio del territorio, Franco Angeli Ed., Milano, pp. 121-145.

Massari, G.; Massari, I. (1980). Risanamento igienico dei locali umidi, Hopeli, Roma, pp. 149-306.

Mattia, F. De; Patarino, E. (2006). Architetture fortificate di età aragonese in Puglia, Capitanata, Terra di Bari e Terra d'Otranto, Mario Adda Ed., Bari, pp. 193-200.

Mora, P.; Mora, L. (1984). "Le superfici architettoniche, materiale e colore”, in Il colore dell'edilizia storica. Riflessioni e ricerche sugli intonaci e le coloriture, Ministero per i Beni Culturali e Ambientali, Roma, pp. 17-24.

Motta, E. (1993). Sulla valutazione della spinta attiva in terrapieni di altezza finita, A.G.I., Roma, pp. 236-249.

Sthamer, E. (1995). L'amministrazione dei castelli nel Regno di Sicilia sotto Federico II e Carlo I d'Angiò, Mario Adda Ed., Bari., pp. 94-150.

Torsello, B.P.; Musso, F. (2003). Tecniche di restauro architettonico, Utet Ed., Torino, pp. 600-663. 